CARDIOVASCULAR MEDICINE

\title{
Effects of digoxin on left atrial function in heart failure
}

\author{
J M Dernellis, M P Panaretou
}

Heart 2003;89:1308-1315

\begin{abstract}
Objective: To investigate the effects of digoxin on left atrial (LA) function in patients with congestive heart failure and dilated left atria.

Patients: 30 patients with enlarged left atrium (maximum LA diameter $>4 \mathrm{~mm}$ ) caused by heart failure (New York Heart Association functional class III or IV) were studied before and after treatment with digoxin (0.25 mg orally for 12 days). Digoxin was also administered to 30 normal participants who served as controls.

Main outcome measures: LA active (AEF) and passive emptying fractions (PEF), reservoir fraction (RF), kinetic energy (KE), and mean velocity of circumferential atrial fibre shortening (Vcf) were calculated from echocardiographic measurements of LA volumes and transmitral Doppler flow velocities at baseline and on the third, sixth, eighth, and 12th day after digoxin administration. Results: LA AEF, PEF, RF, KE, and Vcf were significantly lower in patients than in controls ( $p<0.001)$. LA $\mathrm{AEF}, \mathrm{PEF}, \mathrm{RF}, \mathrm{KE}$, and $\mathrm{Vcf}$ increased significantly both in patients and controls after digoxin administration $(p<0.001)$. This increase was greater in patients than in controls $(p<0.001)$. KE was linearly correlated with LA volume at the onset of atrial systole in all participants. The slope and the intercept of this relation were significantly increased after digoxin both in patients and in controls $(p<0.001$ ).

Conclusions: LA performance is impaired in patients with heart failure. Dilated atria manifest atrial failure. Digoxin improves LA performance and LA contractility both in dilated and in normal atria. The effects of digoxin on LA contractility are augmented in the failing atria compared with the normal atria.
\end{abstract}

See end of article for authors' affiliations Correspondence to: Dr J M Dernellis, Vostanio Hospital, 1 Kathigitou Street, Mytilene 81100 , Greece; dernellis@ yahoo.gr

Accepted 5 June 2003

....................

$\mathrm{F}$ or more than 200 years, digitalis glycosides have occupied a prominent place in the management of congestive heart failure and certain arrhythmias. ${ }^{1}$ The pharmacological, haemodynamic, and electrophysiological properties of digoxin are well defined. ${ }^{2}$ However, the effects of digoxin on left atrial (LA) function have not been studied. Patients who use digoxin frequently have dilated left ventricles and left atria. ${ }^{3}$ This study was undertaken to investigate the effects of digoxin on LA function in patients with congestive heart failure and dilated left atria and in normal participants with normal left atria.

LA function is characterised by reservoir, conduit, and pump function. ${ }^{4}$ LA pump function can be expressed by the LA active emptying fraction (AEF), kinetic energy (KE), and mean velocity of circumferential atrial fibre shortening (Vcf). All these indices can be measured non-invasively by means of M mode, two dimensional, and Doppler echocardiography. ${ }^{5}$ Recently, we have shown a very good correlation of LA KE with LA stroke work measured invasively from the A loop of the LA pressure-area relation. ${ }^{6}$ Thus, in this study we examined the relation between LA KE and LA volume at the onset of atrial systole, which expresses LA preload, at baseline, and after digoxin administration both in patients with heart failure and in normal participants. This relation represents Starling's law of the left atrium.

\section{METHODS}

\section{Study population}

Thirty patients with congestive heart failure and dilated left atrium (LA diameter from M mode echocardiography $>4 \mathrm{~mm}$ ) who were in sinus rhythm were studied at baseline and after digoxin administration. All patients had left ventricular systolic dysfunction (ejection fraction $<0.40$ ) caused by ischaemic heart disease and they were in stable New York Heart Association (NYHA) functional class III or IV heart failure. Exclusion criteria were age $>80$ years, myocardial infarction within 30 days, and

structural valvar heart disease. Digitalis glycosides were being given to 16 patients and were discontinued for 15 days before baseline measurements. Angiotensin converting enzyme inhibitors and diuretics were being given to all patients and were continued during the study at the same dosage.

Thirty normal age and sex matched participants served as controls and were also studied before and after digoxin administration. Control participants had a normal sized left atrium $(<4 \mathrm{~mm})$ and were all in sinus rhythm. Patients and controls had plasma concentrations of urea and creatinine within normal range. The protocol was approved by the hospital's ethics committee. All participants gave informed written consent.

\section{Study protocol}

All measurements were done with echocardiography at steady state, at $9 \mathrm{am}$ and before digoxin administration. Blood samples were collected before baseline measurements and before the 12th day echocardiographic measurements to examine standard biochemical parameters including plasma urea, creatinine, and electrolytes, as well as serum concentrations of digoxin. After baseline measurements $0.25 \mathrm{mg}$ of digoxin was given orally and the same dose was repeated every day after each study. Echocardiographic measurements were repeated on the third, sixth, ninth, and 12th day.

\footnotetext{
Abbreviations: AEF, active emptying fraction; $d_{a}$ left atrial diameter at the onset of left atrial systole; $d_{\min }$, left atrial end systolic diameter; $E P$, left atrial ejection period; KE, kinetic energy; LA, left atrial; NYHA, New York Heart Association; PEF, passive emptying fraction; RF, reservoir fraction; $V_{a}$, left atrial volume at the onset of atrial systole; $V c f$, mean velocity of circumferential atrial fibre shortening; $V_{\max }$, maximum left atrial volume; $\mathrm{V}_{\min }$, minimum left atrial volume
} 


\section{Echocardiographic measurements}

Routine M mode, two dimensional, and Doppler echocardiograms were recorded for all patients. LA diameters and volumes and transmitral blood flow velocities were obtained simultaneously with the ECG during a steady state with commercially available equipment (Image Point, Hewlett Packard, Andover, Massachusetts, USA) with a $2.5 \mathrm{MHz}$ transducer by the same expert operator.

LA M mode echocardiography was carried out by directing the $M$ mode echo beam to the aortic root where the LA dimension was obtained (fig 1).

A standard apical four chamber view was recorded first and then the heart was imaged in an apical long axis view by rotating the transducer $90^{\circ}$ clockwise to obtain a view as perpendicular as possible to the four chamber view. The transducer was carefully angled to qualitatively maximise LA size, and gain positions were adjusted to obtain the clearest outline of the endocardium. LA measurements were obtained from two dimensional echograms with the largest LA size at the end of ventricular systole. The area of the left atrium was planed (fig 2). Special attention was focused on tracings of chamber images. Thus, if the atrial septum partially dropped out, its location was approximated by reconstructing its image from visualised fragments. Moreover, instead of tracing along the inner surface of the coapted mitral valve, a straight line connecting both sides of the leaflet base attachment points to the valve ring was taken as the border between ventricle and atrium. Both the atrial appendage and the pulmonary veins, when visualised, were carefully excluded. $^{7}$

To quantify left ventricular volume, the endocardium is traced in both systole and diastole. End systole was defined as the last frame before mitral opening with end diastole just before valve closure. The biplane method of discs was used from the apical four chamber view. Special attention was focused on displaying the true left ventricular apex, showing neither the aorta nor the coronary sinus while maximising right ventricular size. ${ }^{8}$

Pulsed wave Doppler measurements were obtained by placing the sample volume at the tip of the mitral valve leaflets to obtain the highest velocity. Colour flow imaging was used to obtain a beam direction as parallel as possible to mitral flow. Five consecutive mitral flow velocity curves were analysed for the derivation of the peak flow velocity of left ventricular early and late diastolic filling ( $\mathrm{E}$ and $\mathrm{A}$ waves). Recordings were made at steady state during end

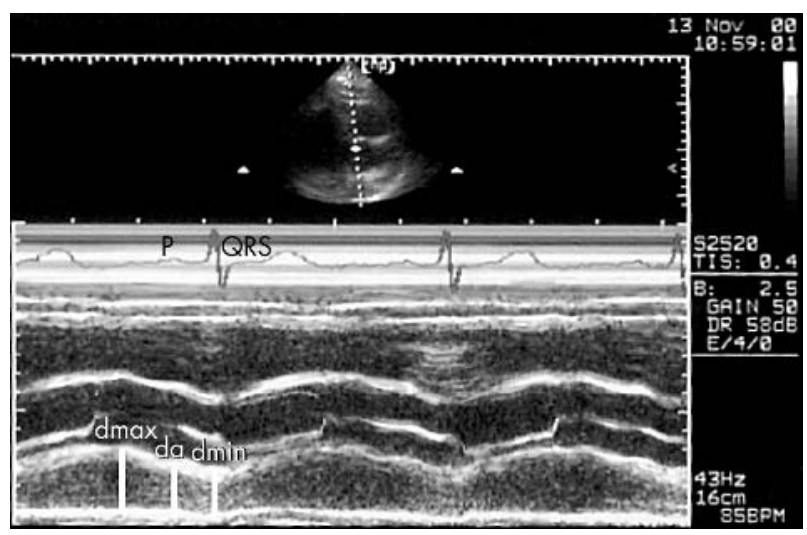

Figure 1 Simultaneous recordings of $M$ mode echocardiography of the left atrium (LA) and ECG. The maximum LA diameter $\left(d_{\text {max }}\right)$, the LA diameter at the onset of LA systole measured at the peak of the $P$ wave of the simultaneously recorded ECG $\left(\mathrm{d}_{\mathrm{a}}\right)$, and the LA end systolic diameter $\left(d_{\min }\right)$ were measured. expiration apnoea with the position of the transducer on the thoracic wall held as constant as possible during the entire procedure.

\section{LA function assessment \\ LA volumes}

From the two dimensional echocardiography LA volume was calculated as $8 / 3 \pi \mathrm{L} \times \mathrm{A}_{1} \times \mathrm{A}_{2}$, where $\pi$ is $3.14, \mathrm{~A}_{1}$ is the LA area in the frontal plane, $A_{2}$ is the LA area in the lateral plane, and $\mathrm{L}$ is the shorter of the long diameters in both planes (fig 2). ${ }^{4} 7910$

\section{LA emptying fractions}

The LA volume at the onset of LA systole measured at the peak of the $\mathrm{P}$ wave of the simultaneously recorded ECG $\left(\mathrm{V}_{\mathrm{a}}\right)$ and the LA end systolic volume $\left(\mathrm{V}_{\min }\right)$ were used to compute LA AEF, which was defined as $\left(V_{a}-V_{\min }\right) / V_{a}$. The maximum LA volume $\left(\mathrm{V}_{\max }\right)$ was used to compute the LA passive emptying fraction (PEF), which was defined as $V_{\max }-V_{a}$ )/ $\mathrm{V}_{\max } \cdot{ }^{4} 71011$

\section{LA reservoir fraction}

The LA reservoir function was measured by the reservoir fraction (RF), which was defined as the $\left.V_{\max }-V_{\min }\right) / V_{\min } \cdot{ }^{1-13}$

\section{Vof}

LA diameter at the onset of LA systole $\left(\mathrm{d}_{\mathrm{a}}\right)$, LA end systolic diameter $\left(\mathrm{d}_{\min }\right)$, and the LA ejection period (EP) were used to compute LA Vcf, which was defined as $\left(\mathrm{d}_{\mathrm{a}}-\mathrm{d}_{\min }\right) /$ $\left(d_{a} \times\right.$ EP) (fig 1$)$. EP was measured as the duration of the A wave of the transmitral Doppler flow velocity. ${ }^{5} 14$

\section{LA KE}

LA KE was obtained from the formula $1 / 2 \cdot \mathrm{m} \cdot \mathrm{v}^{2}$, where $\mathrm{m}=($ LA stroke volume $) \cdot \rho\left(\rho=1.06 \mathrm{~g} / \mathrm{cm}^{3}\right.$, blood's density) and $\mathrm{V}$ is transmitral Doppler A velocity. LA stroke volume was calculated as $\mathrm{V}_{\mathrm{a}}-\mathrm{V}_{\min }$. Where $\mathrm{E}$ and $\mathrm{A}$ waves were not completely separate, the transmitral velocity (v) was defined as the perpendicular difference between the crossover point and the peak of the A wave (fig 3). ${ }^{8} 10$

\section{Statistical analyses}

Data are expressed as mean (SD). The Bonferroni test was used to compare the variables between patients and controls at baseline. Repeated measures analysis of variance was used to compare changes in controls and patients over time. Repeated measures analysis of variance with group as a factor was used to compare the changes between the groups (controls and patients). The general linear model-general factorial procedure, with LA KE the dependent variable and $\mathrm{V}_{\mathrm{a}}$ independent variable, and time as a factor was used to test the differences between the regression lines. To test the effects and interactions of the changes in left ventricular size and function on the changes in LA size and function, repeated measures analysis of covariance was used with LA parameters as dependent variables, and end diastolic and end systolic left ventricular volume indices and left ventricular ejection fraction as covariates. The level of significance was $\mathrm{p}<0.05$.

\section{RESULTS}

On the 12th day, the average serum digoxin concentration was $0.96(0.32) \mathrm{ng} / \mathrm{ml}$ (range $0.6-1.4 \mathrm{ng} / \mathrm{ml}$ ) in the group of patients and $1.04(0.03) \mathrm{ng} / \mathrm{ml}$ (range $0.6-1.4 \mathrm{ng} / \mathrm{ml}$ ) in the group of controls.

\section{Coefficient of variability}

The intraobserver variability was quantified by calculation of a coefficient of variability, defined as the standard deviation 


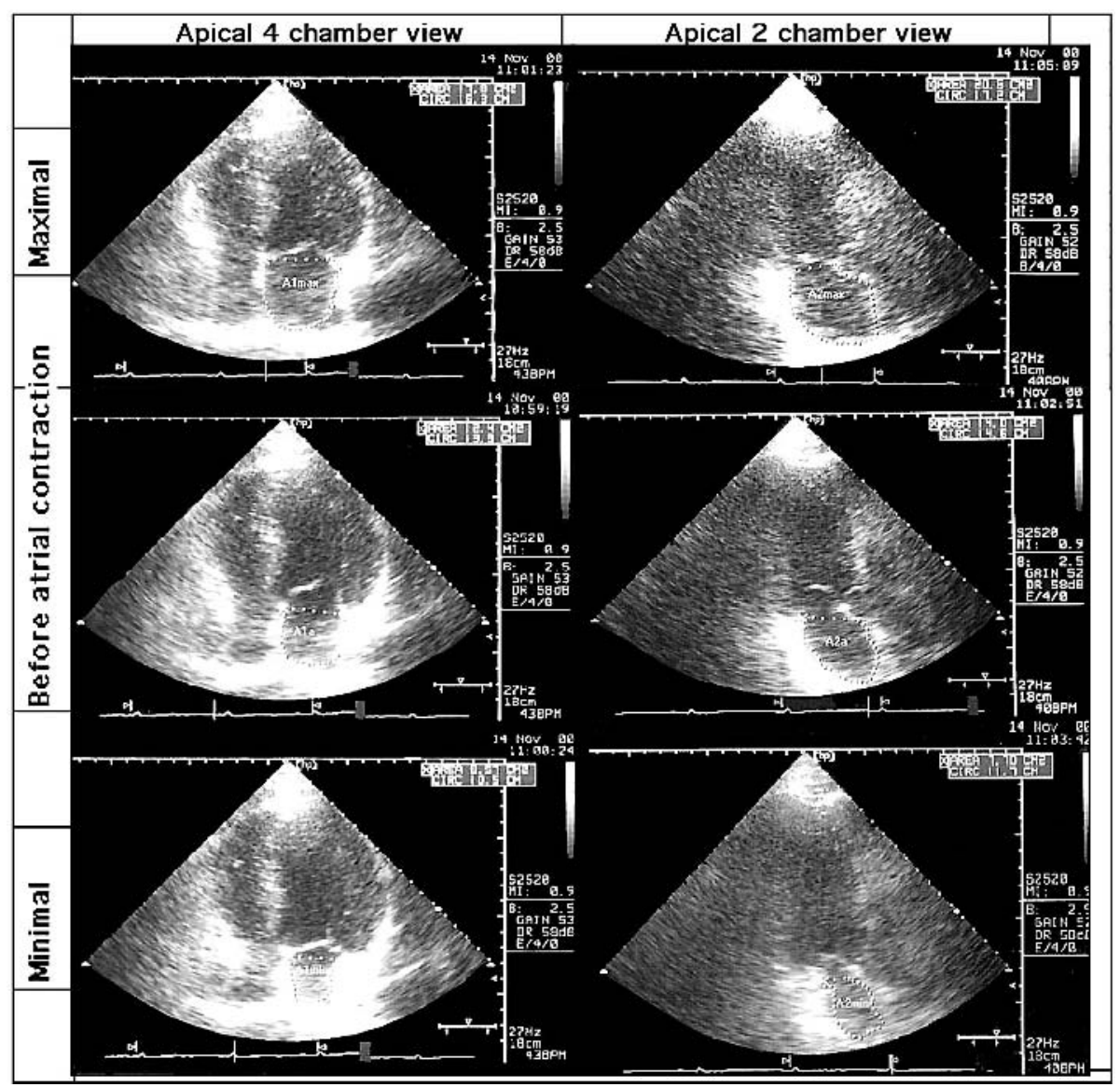

Figure 2 LA area measured in the apical four and two chamber views by means of two dimensional echocardiography. The maximum, minimum, and area before LA contraction measured at the peak of the $\mathrm{P}$ wave of the simultaneously recorded ECG are shown.

of the difference between paired measurements from 20 consecutive participants ( 10 patients and 10 controls) divided by the average value of the mean of each set of the consecutive measurements. The mean percentage intraobserver variability in the measurements of LA AEF, PEF, RF, KE, Vcf, and digoxin were $6.8 \%, 7.3 \%, 5.2 \%, 7.9 \%, 8.0 \%$, and $7.3 \%$, respectively.

\section{Comparisons between patients and controls at baseline}

There was no difference in age (controls 67 (4) years and patients 68 (5) years) and in sex (controls 18 men, 12

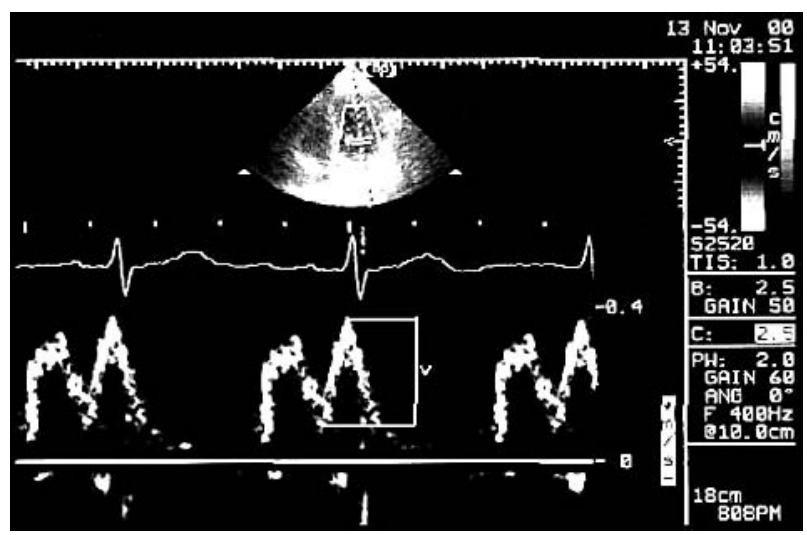

Figure 3 Calculation of blood flow velocity (v) during atrial systole when $E$ and $A$ waves were not completely separated. The cross over point was used because blood was assumed to be accelerated from this point and not from the baseline. women; patients 19 men, 11 women) between the two groups. Patients with heart failure had significantly increased heart rate and decreased blood pressure compared with controls. LA volumes $\left(\mathrm{V}_{\max }, \mathrm{V}_{\mathrm{a}}\right.$, and $\left.\mathrm{V}_{\min }\right)$ were significantly increased in heart failure. AEF, PEF, RF, peak velocity of blood flow during atrial systole, KE, and LA Vcf were all significantly decreased in heart failure atria. Finally, the slope and the intercept were also decreased in patients with heart failure. All comparisons were significant at $\mathrm{p}<0.001$ except for diastolic blood pressure, which was significant at $\mathrm{p}=0.002($ tables 1 and 2$)$.

\section{Haemodynamic effects of digoxin}

Heart rate decreased progressively during digoxin administration in patients $(\mathrm{p}<0.001)$ but did not change in controls (not significant; tables 1 and 2). Conversely, blood pressure did not change in patients, but systolic and diastolic blood pressure increased in controls. This increase reached a significant level for diastolic blood pressure $(\mathrm{p}=0.002)$.

\section{Effects of digoxin on LA function}

LA volumes measured at three different times $\left(\mathrm{V}_{\max }, \mathrm{V}_{\mathrm{a}}\right.$, and $\mathrm{V}_{\min }$ ) were significantly reduced in patients after digoxin administration $(\mathrm{p}<0.001)$, but in controls only $\mathrm{V}_{\mathrm{a}}$ $(p=0.025)$ and $V_{\text {min }}(p<0.001)$ were reduced. In controls $\mathrm{V}_{\max }$ did not change. Both in patients and in controls PEF and AEF were significantly increased over time during digoxin administration $(\mathrm{p}<0.001$ for all comparisons). Similarly, transmitral flow velocity during atrial systole increased over time after digoxin administration ( $p=0.007$ in controls and $p<0.001$ in patients). The same was true for KE and Vcf ( $p<0.001)$ (tables 1 and 2, fig 4). In patients with heart failure Vcf was nearly doubled (fig 5). 
Table 1 Haemodynamic data and left atrial (LA) variables at baseline and after digoxin administration in controls $(n=30)$

\begin{tabular}{|c|c|c|c|c|c|c|c|}
\hline & Baseline & 3rd day & 6th day & 9th day & 12th day & P1 & P2 \\
\hline HR (beats/min) & $75(6)$ & $74(5)$ & $74(8)$ & $74(12)$ & $74(16)$ & 0.949 & 0.000 \\
\hline $\mathrm{SBP}(\mathrm{mm} \mathrm{Hg})$ & 109 (10) & $111(8)$ & $112(10)$ & $114(15)$ & $116(22)$ & 0.077 & 0.000 \\
\hline $\mathrm{DBP}(\mathrm{mm} \mathrm{Hg})$ & $69(6)$ & $70(5)$ & $72(6)$ & $74(9)$ & $75(13)$ & 0.002 & 0.002 \\
\hline EDVI $\left(\mathrm{ml} / \mathrm{m}^{2}\right)$ & $53.62(4.91)$ & $53.38(4.33)$ & $53.14(5.83)$ & $52.90(8.34)$ & $52.66(11.21)$ & 0.000 & 0.000 \\
\hline ESVI $\left(\mathrm{ml} / \mathrm{m}^{2}\right)$ & $17.71(1.65)$ & $17.12(1.32)$ & $16.53(1.13)$ & $15.93(1.15)$ & $15.34(1.38)$ & 0.000 & 0.000 \\
\hline LVEF & $0.67(0.05)$ & $0.67(0.03)$ & $0.68(0.03)$ & $0.69(0.04)$ & $0.70(0.06)$ & 0.035 & 0.000 \\
\hline $\mathrm{V}_{\max }(\mathrm{ml})$ & $50.0(5.9)$ & $49.1(3.4)$ & $48.2(4.3)$ & $47.9(7.6)$ & $47.6(11.4)$ & 0.576 & 0.000 \\
\hline $\mathrm{V}_{\mathrm{a}}(\mathrm{ml})$ & $29.5(3.1)$ & $28.8(2.1)$ & $28.0(2.9)$ & $27.4(4.8)$ & $26.8(6.9)$ & 0.025 & 0.000 \\
\hline$V_{\min }(\mathrm{ml})$ & $17.8(2.5)$ & $16.9(1.5)$ & $16.0(2.0)$ & $15.1(3.3)$ & $14.2(4.9)$ & 0.000 & 0.000 \\
\hline PEF & $0.41(0.03)$ & $0.41(0.02)$ & $0.42(0.03)$ & $0.43(0.05)$ & $0.44(0.07)$ & 0.025 & 0.000 \\
\hline AEF & $0.40(0.04)$ & $0.41(0.03)$ & $0.43(0.04)$ & $0.45(0.07)$ & $0.47(0.10)$ & 0.000 & 0.000 \\
\hline RF & $1.82(0.26)$ & $1.89(0.20)$ & $1.99(0.27)$ & $2.17(0.50)$ & $2.45(0.99)$ & 0.000 & 0.000 \\
\hline $\mathrm{v}(\mathrm{cm} / \mathrm{s})$ & $37.2(6.3)$ & $38.0(4.6)$ & $39.0(6.3)$ & $40.8(10.6)$ & $42.6(15.5)$ & 0.007 & 0.000 \\
\hline KE (dynes.cm) & 9039 (3843) & 9369 (2957) & $10278(4315)$ & 13094 (8847) & 15910 (13 995) & 0.000 & 0.000 \\
\hline LA Vcf $(/ s)$ & $1.24(0.19)$ & $1.30(0.13)$ & $1.39(0.18)$ & $1.52(0.34)$ & $1.64(0.53)$ & 0.000 & 0.000 \\
\hline Slope (dynes $/ \mathrm{cm}^{2}$ ) & 778 & 1003 & 1065 & 1278 & 1378 & & \\
\hline Intercept (dynes $\cdot \mathrm{cm})$ & 13903 & 19475 & 19515 & 20987 & 21871 & 0.000 & \\
\hline$r$ & 0.63 & 0.71 & 0.72 & 0.69 & 0.68 & & \\
\hline
\end{tabular}

AEF, active emptying fraction; DBP, diastolic blood pressure; EDVI, end diastolic left ventricular volume index; HR, heart rate; ESVI, end diastolic left ventricular volume index; KE, kinetic energy; LVEF, left ventricular ejection fraction; P1, comparisons of patients over time (repeated measures analysis of variance); P2 comparisons between controls and patients over time (repeated measures analysis of variance); PEF, passive emptying fraction; RF, reservoir fraction; SBP, systolic blood pressure; $v$, peak velocity of blood flow during atrial systole; $V_{a}$, left atrial volume at the onset of atrial systole; $V_{\text {max }}$ maximum left atrial volume; $V_{\text {min, }}$ minimum left atrial volume; Vcf, mean velocity of circumferential atrial fibre shortening.

Moreover, RF was significantly increased both in patients and in controls $(\mathrm{p}<0.001)$ (tables 1 and 2 , fig 4$)$.

\section{Left ventricular function}

Digoxin significantly decreased left ventricular end diastolic and end systolic volumes in patients and controls $(\mathrm{p}<0.001)$ (tables 1 and 2$)$. Left ventricular ejection fraction was significantly increased in patients $(\mathrm{p}<0.001)$ and controls $(p<0.035)$. Changes in size and in left ventricular ejection fraction were greater in patients than in controls (table 2). End diastolic and end systolic volume indices and left ventricular ejection fraction had no significant effect on LA volumes and LA function indices (PEF, AEF, RF, KE, and LA Vcf) when left ventricular parameters were entered as covariates in repeated measures analysis of covariance models.

\section{Comparisons within patients}

Patients were divided in two groups: group A consisted of 16 patients restarting digoxin after a short break, and group B consisted of the remaining 14 patients starting digoxin for the first time. There was no difference between the two groups concerning haemodynamic, left ventricular, and LA variables at baseline. Repeated measures analysis of variance with group (A or B) as the between participants factor showed that KE and Vcf were significantly different between the two groups over time $(p<0.001$ and $p=0.002$, respectively). All other variables were not significantly different between the two groups.

\section{Significant correlations}

A very good correlation was found between $K E$ and $V_{a}$ in controls and in patients at baseline and after digoxin administration (table 3). As KE represents LA stroke work and $V_{\mathrm{a}}$ represents LA preload, this relation represents the LA Frank-Starling law. The slope and the intercept of this linear relation were increased during digoxin administration $(\mathrm{p}<0.001)$ (tables 1 and 2, fig 6).

There was also a very good correlation between serum concentrations of digoxin and LA KE on the 12th day in

Table 2 Haemodynamic data and LA variables at baseline and after digoxin administration in patients with heart failure $(n=30)$

\begin{tabular}{|c|c|c|c|c|c|c|c|}
\hline & Baseline & 3rd day & 6th day & 9th day & 12th day & Pl & P2 \\
\hline HR (beats/min) & $84(5)$ & $83(5)$ & $82(5)$ & $82(5)$ & $81(5)$ & 0.000 & 0.000 \\
\hline $\mathrm{SBP}(\mathrm{mm} \mathrm{Hg})$ & $99(6)$ & $101(6)$ & $100(6)$ & 100 (7) & $99(6)$ & 0.819 & 0.000 \\
\hline $\mathrm{DBP}(\mathrm{mm} \mathrm{Hg})$ & $65(5)$ & $65(4)$ & $65(4)$ & $65(4)$ & $65(5)$ & 0.983 & 0.000 \\
\hline EDVI $\left(\mathrm{ml} / \mathrm{m}^{2}\right)$ & $159.71(12.66)$ & $146.72(9.10)$ & $133.73(7.27)$ & 120.74 (8.37) & $107.75(11.61)$ & 0.000 & 0.000 \\
\hline ESVI $\left(\mathrm{ml} / \mathrm{m}^{2}\right)$ & $104.33(8.23)$ & 93.05 (5.99) & $81.77(4.29)$ & $70.50(3.90)$ & $59.22(5.13)$ & 0.000 & 0.000 \\
\hline LVEF & $0.34(0.05)$ & $0.37(0.04)$ & $0.40(0.04)$ & $0.42(0.04)$ & $0.45(0.05)$ & 0.000 & 0.000 \\
\hline$V_{\max }(\mathrm{ml})$ & $101.6(10.5)$ & $99.6(10.3)$ & $97.6(10.1)$ & $95.6(10.0)$ & $93.7(9.8)$ & 0.000 & 0.000 \\
\hline $\mathrm{Va}(\mathrm{ml})$ & $76.5(8.7)$ & $72.4(8.4)$ & $68.4(8.1)$ & $64.2(7.9)$ & $59.8(7.7)$ & 0.000 & 0.000 \\
\hline Vmin (ml) & $65.0(7.5)$ & $60.0(7.0)$ & $55.0(6.5)$ & $49.9(6.1)$ & $44.7(5.7)$ & 0.000 & 0.000 \\
\hline PEF & $0.25(0.02)$ & $0.27(0.02)$ & $0.30(0.02)$ & $0.33(0.03)$ & $0.36(0.03)$ & 0.000 & 0.000 \\
\hline AEF & $0.15(0.01)$ & $0.17(0.01)$ & $0.20(0.02)$ & $0.22(0.02)$ & $0.25(0.02)$ & 0.000 & 0.000 \\
\hline $\mathrm{RF}$ & $0.56(0.05)$ & $0.65(0.06)$ & $0.77(0.07)$ & $0.90(0.09)$ & $1.08(0.13)$ & 0.000 & 0.000 \\
\hline$v(\mathrm{~cm} / \mathrm{s})$ & 22.8 (3.9) & $24.0(4.1)$ & $29.1(5.1)$ & $31.0(5.5)$ & $32.5(5.8)$ & 0.000 & 0.028 \\
\hline KE (dynes.cm) & $3361(1740)$ & $4051(2060)$ & 6474 (3395) & $7819(4031)$ & 9131 (4583) & 0.000 & 0.000 \\
\hline LA Vcf $(/ s)$ & $0.42(0.06)$ & $0.49(0.07)$ & $0.58(0.08)$ & $0.68(0.10)$ & $0.80(0.11)$ & 0.000 & 0.000 \\
\hline Slope (dynes $/ \mathrm{cm}^{2}$ ) & 113 & 148 & 273 & 351 & 445 & & \\
\hline Intercept (dynes.cm) & 5250 & 6682 & 12213 & 14694 & 17484 & 0.000 & \\
\hline$r$ & 0.57 & 0.61 & 0.66 & 0.69 & 0.75 & & \\
\hline
\end{tabular}



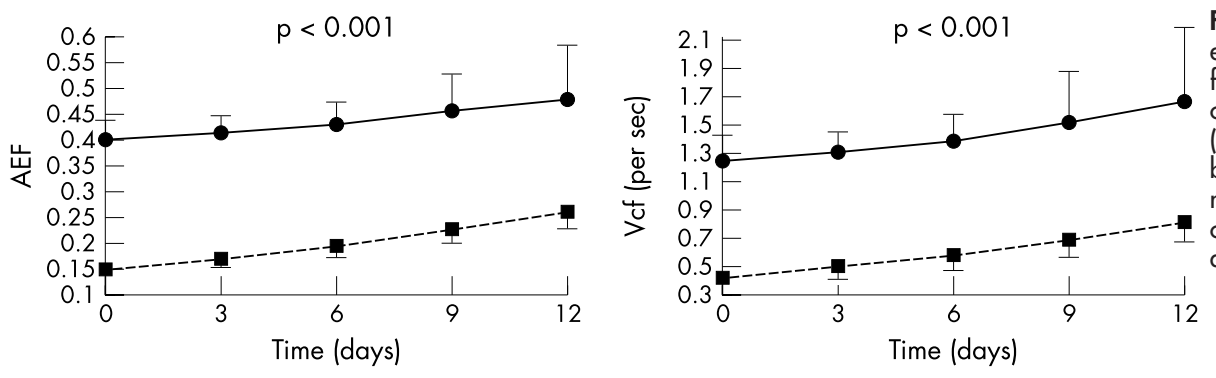

Figure 4 Mean (SD) value of active emptying fraction (AEF), reservoir fraction (RF), mean velocity of circumferential atrial fibre shortening (Vcf), and kinetic energy (KE) at baseline and during the third, sixth, ninth, and 12th day in patients and controls show a gradual increase after digoxin administration.
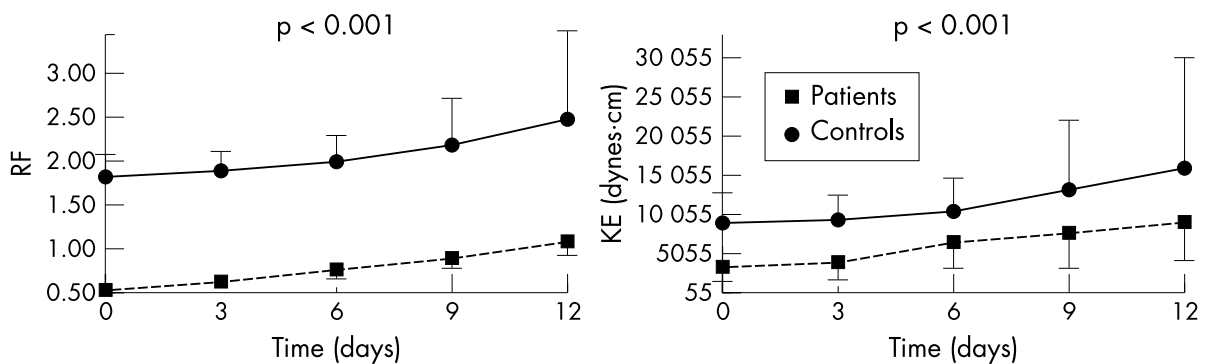

controls $(r=0.97, \mathrm{p}=0.0001)$ and in patients $(r=0.91$, $\mathrm{p}=0.0001)$. Vcf and AEF were also correlated with serum concentrations of digoxin but there was no correlation between PEF, RF, and serum concentrations of digoxin (table 3). The high levels of the correlation coefficient between serum concentrations of digoxin and LA KE are

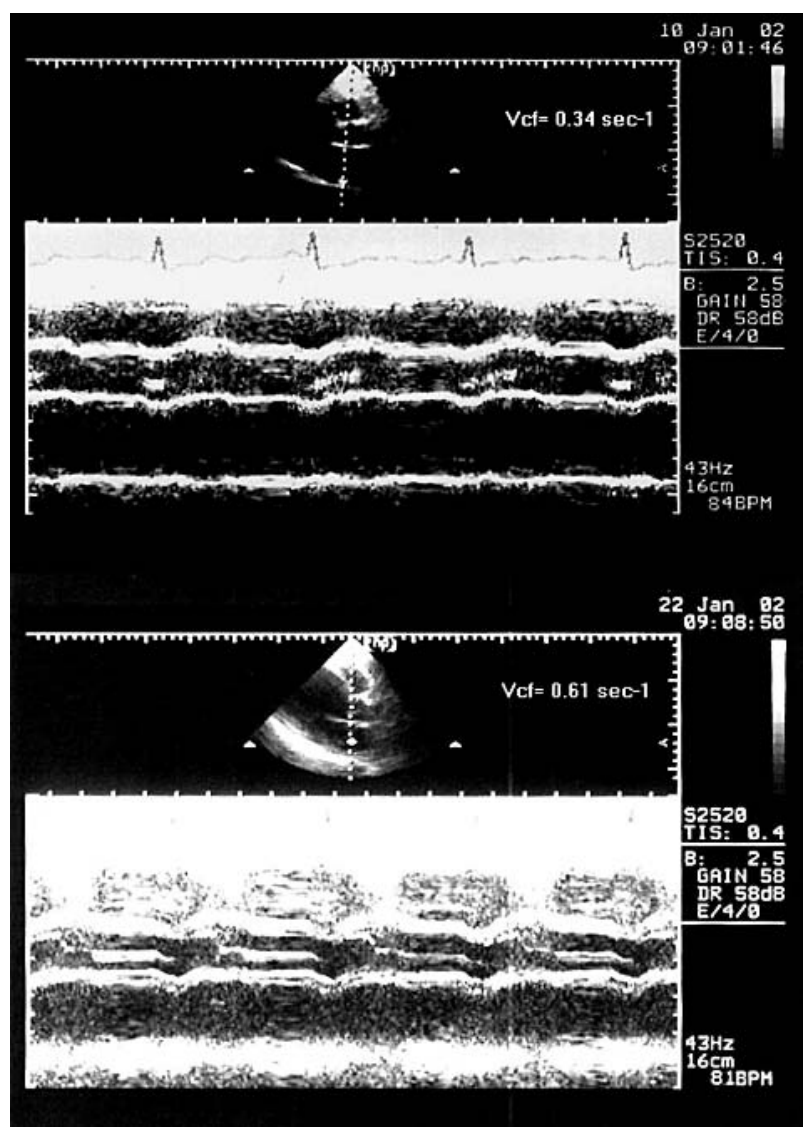

Figure 5 Representative $M$ mode echocardiograms showing the increase in Vcf after digoxin administration in dilated left atria compared with baseline. Vcf is the ratio of LA fractional shortening during atrial contraction (M mode) to the LA ejection period (Doppler). explained by the simultaneous echocardiographic determinations and blood analysis 24 hours after the last digoxin administration and by the high levels of reproducibility of the two variables.

\section{DISCUSSION}

The main findings of this study are as follows. PEF, AEF, and LA systolic velocity are reduced in patients with heart failure. An increased inotropic state after digoxin administration resulted in improved LA emptying function and systolic velocity both in normal participants and in patients with heart failure. It is important that all these effects of digoxin on LA function be correlated with the serum concentrations of digoxin because it seems that these effects cannot be attributed to improvement in left ventricular function. The relation between LA KE and $\mathrm{V}_{\mathrm{a}}$ may provide a reliable noninvasive determination of Starlings' law of the left atrium. By means of this method, it was found that the LA KE- $\mathrm{V}_{\mathrm{a}}$ relation is highly linear and sensitive to changes in inotropic state.

\section{Methodological considerations}

All control participants in the study had normal renal function and digoxin was not given or discontinued. Treatment concentrations of digoxin were achieved in all patients. No adverse events were observed. This is a solely non-invasive study of LA function with a control group, which received digoxin. KE and Vcf were also calculated except for AEF as measures of LA pump function. KE as a surrogate of LA stroke work was validated in a previous study. ${ }^{6}$ The non-invasive LA Frank-Starling relation was determined for the first time in the present study.

However, there are two limitations of the study. Firstly, atrial function was evaluated in terms of reservoir and pump function. This model is limited, as it did not take into account the conduit function of the atrium and the motion of the atrial base (atrioventricular ring) around the blood. The main purpose of the study was to evaluate the effects of digoxin on LA pump function. Secondly, in patients with heart failure in NYHA class III and IV the left ventricular filling pattern is mainly restrictive. Thus, atrial systole causes retrograde flow in the pulmonary veins and the volume change in the atrium does not represent the mass flowing forward across the mitral valve. Furthermore, peak velocity is achieved for only a very short period. Thus, calculations of $\mathrm{KE}$ are limited 
Table 3 Correlations between digoxin plasma concentrations and LA variables in controls and patients

\begin{tabular}{llllll}
\hline & \multicolumn{2}{l}{ Controls } & & \multicolumn{2}{l}{ Patients } \\
\cline { 2 - 3 } \cline { 5 - 6 } \cline { 5 - 6 } & $\boldsymbol{r}$ & $\mathbf{p}$ Value & & $\boldsymbol{r}$ & p Value \\
\hline PEF & 0.18 & NS & & 0.12 & NS \\
AEF & 0.48 & 0.005 & & 0.55 & 0.002 \\
RF & 0.30 & NS & & 0.09 & NS \\
KE (dynes.cm) & 0.97 & 0.0001 & & 0.91 & 0.0001 \\
Vcf $(/ \mathrm{s})$ & 0.70 & 0.0001 & & 0.67 & 0.0001 \\
\hline
\end{tabular}

NS, not significant

because they assume that the whole mass is accelerated to the peak A wave velocity. However, it has been shown that there is a very good correlation between LA stroke work measured invasively and LA KE in both normal and enlarged atria in patients with congestive heart failure and with mitral stenosis. ${ }^{6}$

\section{LA failure}

We found that in patients with NYHA functional class III or IV heart failure, the left atria were dilated with diminished contractility and decreased LA reservoir function. These findings determine the LA failure syndrome as described previously. ${ }^{11} 1415$

\section{Haemodynamic effects of digoxin administration}

We found an increase of blood pressure in normal participants after digoxin administration, which results from an increase in peripheral arterial resistance due to vasoconstriction caused by digoxin in smooth muscle cells of arterioles. ${ }^{1}{ }^{1617}$ On the other hand in patients with heart failure the heart rate decreased, probably because digoxin improved the clinical syndrome of heart failure, which resulted in decreased sympathetic nervous system activation..$^{2} 31718$

\section{Effects of digoxin on LA function}

$\mathrm{V}_{\max }$ was reduced only in patients with heart failure. In controls it did not change. These results may reflect the correlation of $\mathrm{V}_{\max }$ with LA pressure. ${ }^{19}{ }^{20}$ Digoxin reduces LA pressure in patients with heart failure who have increased LA pressures, but there are no changes in the normal pressures in controls. ${ }^{1-3} 17 \quad \mathrm{~V}_{\mathrm{a}}$ and $\mathrm{V}_{\min }$ were decreased both in patients and in controls because these volumes depend on the LA emptying function. LA AEF and PEF were both increased in patients and controls. Thus, $V_{a}$ and $V_{\text {min }}$ were decreased both in patients and
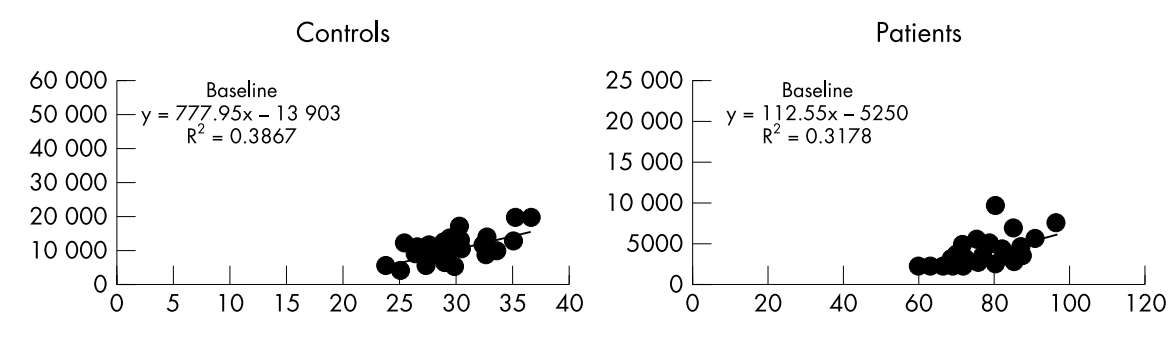

Figure 6 Relation between LA volume at the onset of atrial systole $\left(V_{a}\right)$ and LA kinetic energy in controls and patients. The regression line is shown. In both groups there is an increase in the slope after digoxin administration; however, this increase is greater in heart failure patients than in controls.
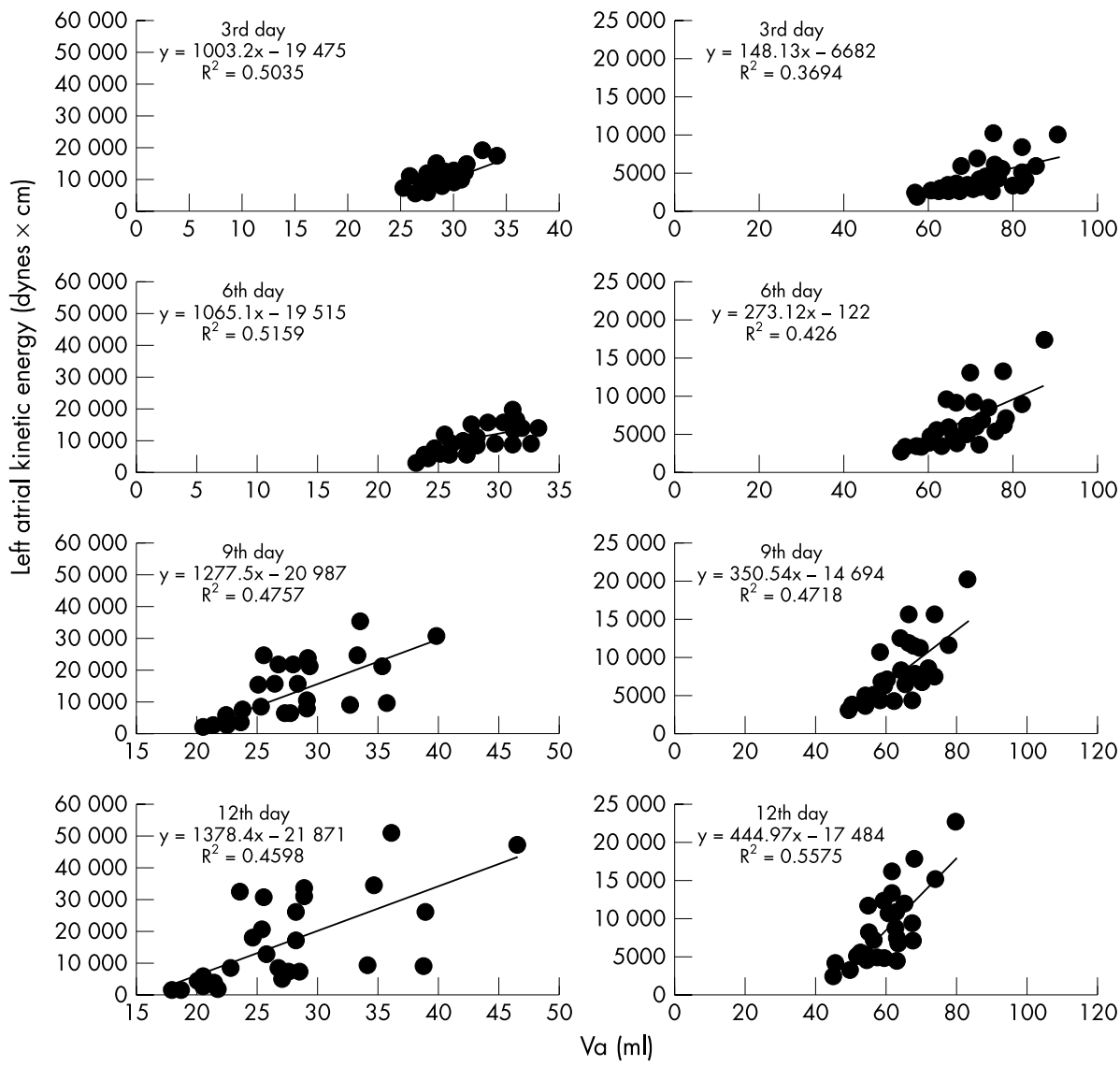
in controls, as digoxin resulted in increased LA emptying. Changes in $\mathrm{V}_{\min }$ were greater than changes in $\mathrm{V}_{\mathrm{a}}$. Therefore, $\mathrm{V}_{\max }$ can be used as a measure of LA volume and pressure while $\mathrm{V}_{\min }$ can be used as a measure of LA emptying function.

Effects of digoxin on LA emptying function are expressed mainly by PEF and AEF. PEF expresses the passive emptying of the left atrium into the left ventricle during early diastole, and it depends on LA and left ventricular diastolic properties and pressures, which in turn rely on left ventricular systole. ${ }^{19}{ }^{20}$ Thus, digoxin may act both directly and indirectly in improving LA passive emptying. AEF expresses LA systole and it is analogous to left ventricular ejection fraction. ${ }^{9-11}$ AEF depends not only on LA systolic function but also on left ventricular end diastole. Left ventricular end diastolic pressure delineates LA afterload. ${ }^{21}{ }^{22}$ Increased AEF may be the beneficial effect of digoxin on left ventricular end diastolic pressure. This is an indirect effect of digoxin on LA pump function.

In this study, systolic function of the left atrium was also assessed by KE and Vcf. Recently, we have shown a very good correlation between KE assessed non-invasively and A loop derived by the LA pressure-area relation in normal and pathological conditions. ${ }^{6}$ Thus, KE may express LA pump function better than AEF. Moreover, Vcf is less load and heart rate dependent than AEF. Vcf also expresses the velocity of LA contraction, as it takes into account the LA ejection period. The incremental effects of digoxin on these two indices may express an increased inotropic effect of digoxin on LA function. This is a direct effect of digoxin on LA pump function.

Another indirect mechanism may be considered. It is well known that unloading the left atrium in patients with restrictive physiology, particularly by using angiotensin converting enzyme inhibitors, results in a dominant atrial filling of the ventricle with a significant increase in blood acceleration during atrial systole. ${ }^{23}$ Thus, as the increase in calculated KE is up to the peak velocity of blood flow during atrial systole, it cannot be excluded that the increase in KE after digoxin administration does not reflect increased contractile performance but is the result of the decreased LA afterload. Finally, restarting digoxin after a short break has a significantly smaller effect on KE and Vcf than starting it for the first time.

LA reservoir function is dependent mainly on LA compliance. ${ }^{24}{ }^{25}$ In a previous study we have shown an increased LA compliance after dobutamine administration in normal study participants and in patients with heart failure. In the present study digoxin had a similar effect on LA reservoir function. Digoxin is also an inotropic agent, which may act on the LA reservoir function indirectly by increasing left ventricular contractility. Whatever the mechanism of digoxin, the increased LA reservoir function is of great clinical importance, as LA reservoir function is directly correlated with cardiac output.

\section{Frank-Starling mechanism of the left atrium}

Cardiac glycoside administration caused the LA function curve (Frank-Starling) to shift upwards and to the left, so that more stroke work would be generated at a given preload $\left(V_{a}\right)$. Both failing and normal atria comply with the FrankStarling law of the heart. A leftward shift of LA KE- $V_{a}$ relation results from the inotropic effect of digoxin on the left atrium. The inotropic effect was observed in both patients and controls. This effect was greater in patients with heart failure than in normal participants because in patients the slope was nearly quadrupled on the 12 th day and in controls it was nearly doubled.

\section{Clinical implications}

The beneficial effects of digoxin on the left atrium in patients with heart failure are obvious. Digoxin decreased LA volume. The regression of LA volume may result in restoration of sinus rhythm, improved LA function, and decreased thrombus formation in the left atrium with concomitant decrease of thromboembolic disease in patients with heart failure and dilated left atrium. Improved LA reservoir function also improves left ventricular filling, which in turn may increase cardiac output in patients with considerable impairment of left ventricular performance.

\section{Conclusions}

Digoxin has an inotropic effect on the left atrium in heart failure and in controls. Digoxin also increases LA reservoir function in heart failure and in controls. These effects may explain in part the beneficial effects of digoxin on the failing and normal heart.

\section{Authors' affiliations \\ J M Dernellis, M P Panaretou, Vostanio Hospital, Mytilene, Greece}

\section{REFERENCES}

1 Hauptman PJ, Kelly RA. Digitalis. Circulation 1999;99:1265-70.

2 Gheorghiade M, Ferguson D. Digoxin: a neurohormonal modulator in heart failure? Circulation 1991;84:2181-6.

3 Gheorghiade M, Hall VB, Jacobsen G, et al. Effects of increasing maintenance dose of digoxin on left ventricular function and neurohormones in patients with chronic heart failure treated with diuretics and angiotensin-converting enzyme inhibitors. Circulation 1995;92:1801-7.

4 Dernellis JM, Vyssoulis GP, Zacharoulis AA, et al. Effects of antihypertensive therapy on left atrial function. J Hum Hypertens 1996; 10:789-94.

5 Stefanadis C, Dernellis J, Toutouzas P. Evaluation of the left atrial performance using acoustic quantification. Echocardiography 1999:16:117-25.

6 Stefanadis C, Dernellis J, Lambrou S, et al. Left atrial energy in normal subjects, in patients with symptomatic mitral stenosis and in patients with advanced heart failure. Am J Cardio 1998;82:1220-3.

7 Dernellis $\mathrm{J}$, Tsiamis E, Stefanadis $\mathrm{C}$, et al. Effects of postural changes on left atrial function in patients with hypertrophic cardiomyopathy. Am Heart $J$ 1998; 136:982-7

8 Silverman NH, Ports TA, Snider AR, et al. Determination of left ventricular volume in children: echocardiographic and angiographic comparisons. Circulation 1980:62:548-57.

9 Stefanadis C, Dernellis J, Toutouzas P. A clinical appraisal of left atrial function. Eur Heart J 2001;22:22-36.

10 Dernellis J, Stefanadis C, Toutouzas P. From science to bedside: the clinical role of atrial function. Eur Heart J 2000;21 (suppl K):K48-57.

11 Stefanadis C, Dernellis J, Stratos C, et al. Assessment of left atrial pressurearea relation in humans by means of retrograde left atrial catheterization and echocardiographic automatic boundary detection: effects of dobutamine. J Am Coll Cardiol 1998:31:426-36.

12 Sanada H, Shimizu M, Sugihara N, et al. Increased left atria chamber stiffness in hypertrophic cardiomyopathy. Br Heart $J$ 1993:69:31-5

13 Grant C, Bunnell IL, Greene DG. The reservoir function of the left atrium during ventricular systole: an angiocardiographic study of atrial stroke volume and work. Am J Med 1964;37:36.

14 Dernellis J, Vyssoulis G, Zacharoulis A, et al. Acute changes of left atrial distensibility in congestive heart failure. Clin Cardiol $1998 \cdot 21 \cdot 28-32$

15 Dernellis J, Stefanadis C, Zacharoulis A, et al. Left atrial mechanical adaptation to long-standing hemodynamic loads based on pressure-volume relations. Am J Cardiol 1998:81:1138-43.

16 Blaustein MP. Endogenous ouabain: role in the pathogenesis of hypertension. Kidney Int 1996;49:1748-53

17 Mason DT, Braunwald E. Studies on digitalis: effects of ouabain on forearm vascular resistance and venous tone in normal subjects and in patients in heart failure. J Clin Invest 1964:43:532-43.

18 Gillis RA, Quest JA. The role of the nervous system in the cardiovascular effects of digitalis. Pharmacol Rev 1980;31:19-97.

19 Appleton CP, Hatle LK, Popp RL. Relation of transmitral flow velocity patterns to left ventricular diastolic function: new insights from a combined hemodynamic and Doppler echocardiographic study. J Am Coll Cardiol 1988;12:426-40.

20 Basnight MA, Gonzalez MS, Kershenovich SC, et al. Pulmonary venous flow velocity: relation to hemodynamics, mitral flow velocity and left atrial volume and ejection fraction. J Am Soc Echocardiogr 1991;4:547-58. 
21 Stefanadis C, Dernellis J Stratos $C$, et al. Effects of balloon mitral valuuloplasty on left atrial function in mitral stenosis as assessed by pressure-area relation. J Am Coll Cardiol 1998;32:159-68.

22 Stefanadis C, Dernellis J, Tsiamis E, et al. Effects of pacing-induced and balloon coronary occlusion ischemia on left atrial function in patients with coronary artery disease. J Am Coll Cardiol 1999;33:687-96.
23 Henein MY, Amadi A, O'Sullivan C, et al. ACE inhibitors unmask incoordinate diastolic wall motion in restrictive left ventricular disease. Heart 1996;76:326-31.

24 Leistad E, Christensen G, llebekk A. Effects of atrial fibrillation on left and right atrial dimensions, pressures, and compliances. Am J Physiol 1993;264:H1093-7.

25 Hoit BD, Walsh RA. Regional atrial distensibility. Am J Physiol 1992;262:H1356-60.

\section{IMAGES IN CARDIOLOGY}

\section{Coronary rupture as a rare cause of acute myocardial ischaemia two months after angioplasty and stenting}

A n 85 year old man first presented with non-ST elevated myocardial infarction. He had refractory ischaemic symptoms. A coronary angiogram done six days after admission showed three vessel disease. Initial balloon angioplasty performed on the culprit proximal left anterior descending artery (LAD) lesion resulted in a type B coronary dissection. Stenting $(3.0 \mathrm{~mm} /$ $16 \mathrm{~mm}$ NIR SOX, Boston Scientific) was then performed. There was distal extension of the dissection, which was covered by a second stent $(3.0 \mathrm{~mm} / 12 \mathrm{~mm}$ S670D AVE, Medtronic), with slight overlap between the two stents. The angiographic result was satisfactory, and the patient was stabilised. He later

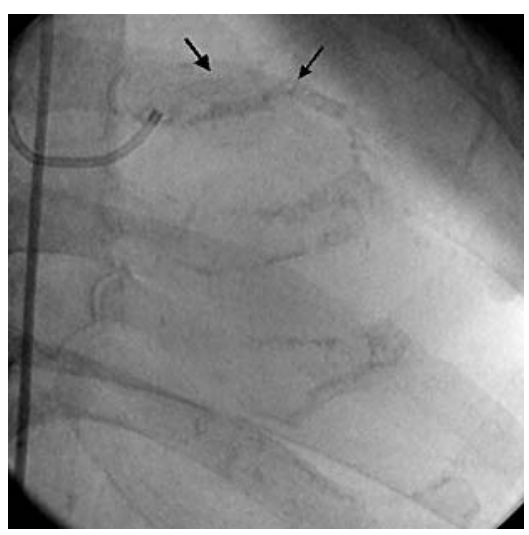

declined second stage angioplasty to other vessels and was discharged. Two months later, he developed acute chest pain and pulmonary oedema. The ECG showed a deep ST depression over all precordial leads. A coronary angiogram was performed, which revealed a ruptured LAD with wide separation of the conjoint ends of the two stents (left panel) and a large coronary pseudoaneurysm (middle panel). On echocardiography (right panel), the large coronary pseudoaneurysm appeared as a well circumscribed cavity in the left anterior aspect of the aortic root and basal anterior region of the left ventricle (LV). Flow into the pseudoaneurysm was demonstrated by colour Doppler.

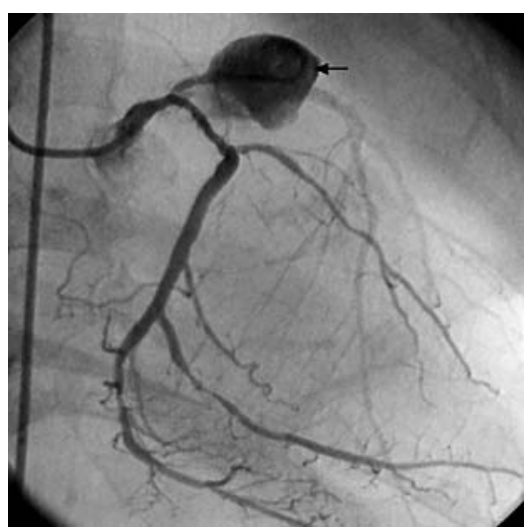

Echocardiography also showed dyskinesia in the anteroseptal and anterior regions (arrowheads), which might have stretched the overlying epicardial coronary artery and ultimately caused the LAD artery rupture at a weak point between the two stents. An attempt was made to deploy a cover stent but a guidewire could not be passed through the pseudoaneurysm down to the distal LAD. In view of the patient's age and other medical co-morbidities, surgery was not contemplated. The patient continued to have refractory heart failure and died four days later.

K-L Tsui

K-H Lam

S-K Li

tsuikin|@netvigator.com

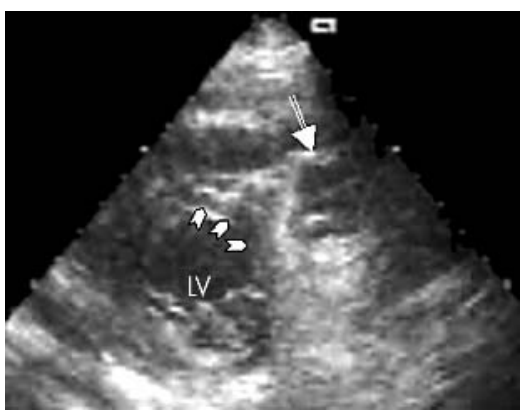

